\title{
Somatic Transformation in Metastatic Testicular Germ Cell Tumours - A Different Disease Entity
}

\author{
ANAND SHARMA $^{1 *}$, CONSTANTINE ALIFRANGIS $^{2,3 *}$, MARINA MILIC $^{1}$, \\ MARCIA HALL ${ }^{1}$, NIKHIL VASDEV ${ }^{4}$, PETER WILSON ${ }^{2}$, ANDREW GOGBASHIAN ${ }^{5}$, \\ DAVID HROUDA $^{6 *}$, DANIEL BERNEY ${ }^{7}$ and JONATHAN SHAMASH ${ }^{2}$ \\ ${ }^{1}$ Department of Medical Oncology, Mount Vernon Cancer Centre, Northwood, U.K.; \\ ${ }^{2}$ Department of Oncology, St Bartholomew's Hospital, London, U.K.; \\ ${ }^{3}$ Department of Oncology, University College London Hospital, London, U.K.; \\ ${ }^{4}$ Department of Urology and Surgery, Lister Hospital, East and North Herts NHS Trust, Stevenage, U.K.; \\ ${ }^{5}$ Department of Radiology, Mount Vernon Cancer Centre, Paul Strickland Scanner Centre, Northwood, U.K.; \\ ${ }^{6}$ Department of Urology, Imperial College Healthcare NHS Trust, Charing Cross Hospital, London, U.K.; \\ ${ }^{7}$ Department of Histopathology, Barts Cancer Institute, London, U.K.
}

\begin{abstract}
Background/Aim: The occurrence of somatic transformation in germ cell tumour (GCT) is rare, with increased incidence in teratomatous tumours. The aim of this study was to understand the clinical outcomes of patients with metastatic GCT with somatic transformation. Materials and Methods: A retrospective study was conducted in two tertiary cancer centres in London. Between 1998 and 2016, 30 cases of somatic transformation in GCT treated at the Mount Vernon Cancer Centre and St. Bartholomew's Hospital were identified. The median age at diagnosis was 34 years (range $=18-56$ years). The histological diagnosis at transformation was rhabdomyosarcoma, sarcomatoid yolk sac, sarcoma (non-specified), clear cell carcinoma, adenocarcinoma and primitive neuro ectodermal tumour (PNET). Results: The 5-year survival rate of all patients was $47 \%$, and that of patients with testicular primary $(n=26$ patients) was $37 \%$. Conclusion: Somatic transformation component in testicular GCTs is generally considered to be an adverse prognostic factor, however, a reasonable 5-year
\end{abstract}

\footnotetext{
*These Authors share first co-authorship.

Correspondence to: Dr. Anand Sharma, MBBS, MD, DPhil, MRCP UK, MRCP Medical Oncology, Consultant Medical Oncologist, Department of Medical Oncology, Mount Vernon Cancer Centre, Northwood, HA6 2RN, U.K. Tel: +44 02038262431, e-mailanand.sharma3@nhs.net; Dr. Constantine Alifrangis, MRCP Ph.D., Consultant Medical Oncologist, Department of Medical Oncology, University College London, 250 Euston road, NW1 2PQ, London, U.K. Tel: +44 07974262388, e-mail: Constantine.alifrangis1@nhs.net

Key Words: Germ cell tumour, rhabdomyosarcoma, sarcoma, immature teratoma, somatic transformation.
}

overall survival rate (87.5\%) was observed in patients who present with this at first diagnosis.

Germ cell tumours most commonly occur in young men and most frequently arise from the testicles. The use of surgery and chemotherapy has been refined over time with the 5-year survival for metastatic disease reaching $80-95 \%(1,2)$. Treatment of metastatic GCT depends on the histological subtype and disease burden, and curative chemotherapy regimens are usually based on cisplatin-based combinations such as BEP (3-5) (bleomycin, etoposide, cisplatin). Infrequently GCT can undergo somatic transformation. This is reported in approximately $3-6 \%$ of these tumours and has been documented as primary presentation or relapse $(6,7)$. These have been reported to be resistant to current chemotherapeutic agents with a relatively poor outlook. The most frequent histological subtypes seen in somatic transformation are rhabdomyosarcoma, primitive neuroectodermal tumour (PNET), adenocarcinoma, neuroblastoma and rarely squamous cell carcinoma (7-11). More recently, some sarcomatoid transformations, have been found to be probably derived from yolk sac tumours and are termed sarcomatoid yolk sac tumours $(12,13)$.

Since the molecular pathogenesis and prognostic factors of sarcomatous transformation are not well understood, its prognosis and significance remain unclear. Because of the rarity of this presentation, uncertainty exists regarding optimal treatment and prognosis. However, a small number of patients have experienced prolonged survival with combined chemotherapy and surgery or radiation (14-16). In the present study, we discuss our experience of somatic transformation of GCTs that were treated at Mount Vernon cancer centre and St Bartholomew's hospital London and discuss the morphologic and clinical outcomes of these rare 
Table I. Patient characteristics and treatment.

\begin{tabular}{lcc}
\hline Histology on transformation $(\mathrm{n}=30)$ & Chemotherapy regime 1 st line & Chemotherapy regime 2nd line \\
\hline Sarcoma $=7$ & BEP $=22$ & GAMEC $=4$ \\
Rhabdomyosarcoma $=9$ & GAMEC $=3$ & VIDE $=2$ \\
PNET $=10$ & POMB $/ \mathrm{ACE}=1$ & $\mathrm{IPO}=5$ \\
Clear cell $=1$ & GEM-TIP $=1$ & GAMMA $=4$ \\
Adenocarcinoma $=2$ & VIP $=1$ & VIDE $=2$ \\
Nephroblastoma $=1$ & EBCa $=1$ & IVAD $=1$ \\
& Cisplatin/ Doxorubicin/ Ifosfamide $=1$ & GEM-TIP $=2$ \\
& & BOP $=1$
\end{tabular}

BEP: Bleomycin/etoposide/cisplatin; GAMEC: granulocyte colony stimulating factor, actiniomycin-D, methotrexate, etoposide, cisplatin; POMB/ACE: cisplatin, vincristine, methotrexate, bleomycin/ actinomycin-D, cyclophosphamide, etopsoide; GEM-TIP: gemcitabine-paclitaxel, ifosfamide, cisplatin; VIP: vincristine, ifosfamide, cisplatin; EBCa: etoposide, bleomycin,cisplatin; VIDE: vincristine, ifosfamide, doxorubicin, etoposide; IVAD: ifosfamide, vincristine, actinomycin-D, doxorubicin; BOP: bleomycin, vincristine, cisplatin.

entities. We also compared those who were transformed to metastatic disease post chemotherapy with those who had viable germ cell tumours - without somatic transformation post chemotherapy.

\section{Patients and Methods}

The medical records of the Mount Vernon Cancer Centre and St Bartholomew's hospital were examined to identify patients with GCT who had somatic transformation. A total of 30 cases were identified in the period of 2000 to 2017. Patients were referred from 6 district general hospitals in the surrounding region, servicing a catchment population of 2.25 million people at Mount Vernon Cancer Centre, and St Bartholomew's referrals for complex and relapsed disease come from the Anglian Germ Cell Cancer Group representing a population of 7.4 million people.

Patients were aged 18 years or older with histologically or cytologically confirmed metastatic testicular cancer, proven metastases on imaging and confirmed somatic transformation, either at presentation or relapse. All new cases are discussed at supra regional multi-disciplinary team meetings where pathological and radiological findings are centrally reviewed and appended to the database by an automated process and updated regularly with clinical follow up data.

\section{Results}

Patient characteristics. A total of 30 patients were identified who had somatic transformation with a diagnosis of GCT. The median age at diagnosis was 34 years (range $=18-56$ years).

We ascertained the presentation timepoint of patients undergoing transformation into three discrete phases of treatment according to when the transformation event occurred; at initial diagnosis, at time of post chemotherapy surgery or at relapse post initial treatment. Of the 30 patients, 9 had somatic transformation at diagnosis, 8 had transformed GCT diagnosed following first line post chemotherapy surgery, and 13 developed it on relapse. The histological diagnoses, treatment type and outcome for each of the 30 patients are detailed in Table I.

Survival. Median overall survival from time of transformation across the cohort was 40 months. The overall survival curve for all patients with somatic transformation is shown in Figure 1. For patients presenting with germ cell tumours and areas of somatic transformation $(n=9)$, median survival was 69 months and the 5-year overall survival was $87.5 \%$. For patients showing residual active disease with somatic transformation following post chemotherapy surgical resection, $(n=8)$ overall survival was 27 months and the 3-year survival was $50 \%$. For patients with transformation at post chemotherapy relapse, median survival was the poorest at 22 months and the 5-year survival was $38.4 \%$ (Tables II and III). Figure 2 shows the univariate mantel-cox analysis for survival of the three discrete groups at which somatic transformation is seen $(p=\mathrm{NS})$.

Chemotherapy regimens. Table I lists the regimens utilised per patient at 1st line, 1st salvage and second salvage chemotherapy, together with the details of any surgical resection performed. Most patients received BEP or dose intensified platinum-based chemotherapy upfront with either the GAMEC or POMB-ACE (filgrastim, actinomycin-D, methotrexate, etoposide, cisplatin and cisplatin, vincristine, methotrexate, bleomycin- actinomycin-D, cyclophosphamide, etoposide respectively) regimens $(15,17)$ with resection of residual disease. At progression or relapse, patients with somatic transformation were treated with second line regimens. Many of these contain cytotoxics with activity in sarcoma such as ifosfamide (present in TIP (cisplatin, ifosfamide, paclitaxel), VIDE (vincristine, ifosfamide, actinomycin-D and etoposide) taxanes [IPO (irinotecan, 


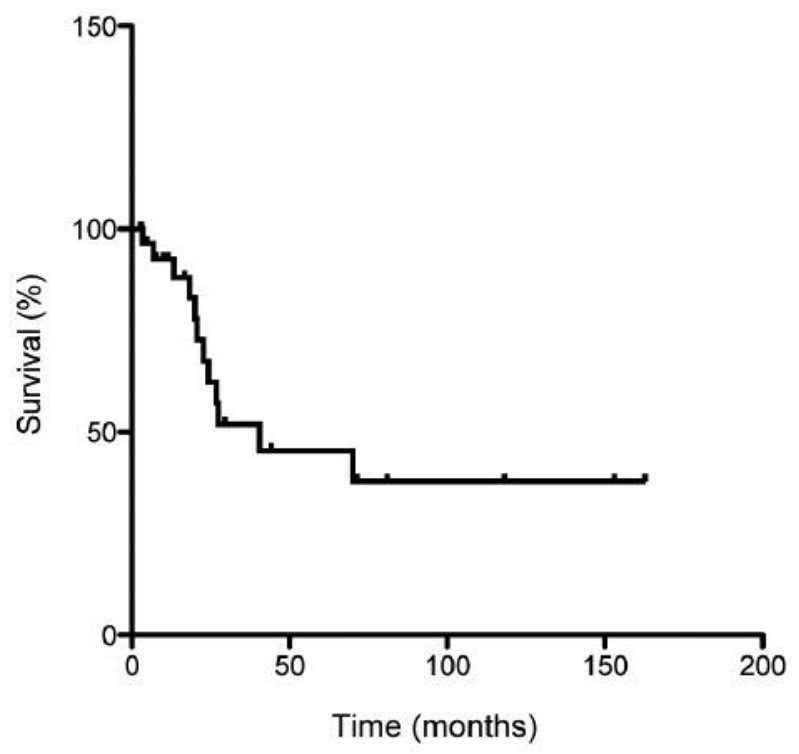

Figure 1. Overall survival in all patients with variant histology. KaplanMeier analysis of overall survival in all patients with variant histology and $\operatorname{GCT}(n=30)$.

Table II. Median survival by timepoint of variant histology diagnosis.

\begin{tabular}{ll}
\hline Number & Median survival (months) \\
\hline $\mathrm{n}=30$ & 40 \\
By presentation with somatic transformation & \\
At Diagnosis ( $\mathrm{n}=9)$ & 69.9 \\
Post chemotherapy surgery (1st line) $(\mathrm{n}=8)$ & 27 \\
at relapse post chemotherapy $(\mathrm{n}=13)$ & 22.7 \\
\hline
\end{tabular}

Table III. Overall survival at 2, 3 and 5 years by timepoint of variant histology diagnosis.

\begin{tabular}{lcc}
\hline Transformation at: & $\mathrm{N}=30$ & OS at 5 years \\
\hline At diagnosis & 9 & $87.50 \%$ \\
Post-chemo & 8 & $50 \%$ \\
At relapse & 13 & $38.40 \%$ \\
All patients & 30 & $47 \%$ \\
Testes & 26 & $62 \% / 39 \% / 31 \%$ \\
Mediastinal & 4 & $50 \%$ \\
\hline
\end{tabular}

paclitaxel, oxaliplatin), TIP] and anthracyclines (cisepirubicin, VIDE). A total of 12 patients underwent high dose chemotherapy as either second- or third-line salvage.

Figure 3 compares the survival outcomes for patients who had viable cancer post primary chemotherapy and surgery,

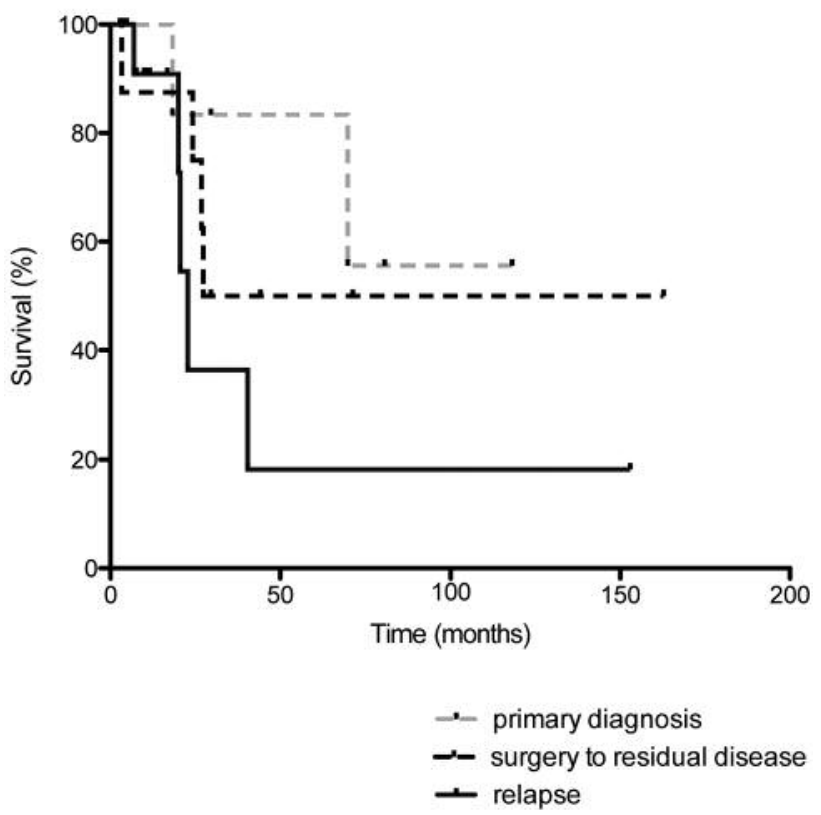

Figure 2. Survival by timepoint at which variant histology is diagnosed. Graph of survival of patients according to whether variant histology is diagnosed at baseline, at post chemotherapy surgery or at relapse.

as shown by histology post-surgery. As has been previously reported, findings of mature teratoma or necrosis only are associated with excellent survival (3-year OS of 86-92\%\%). Conversely, the presence of viable cancer confers a much worse outcome with a 3 -year OS of $40-45 \%$. This is surprisingly very similar whether transformed histology or standard residual germ cell histology is seen.

\section{Discussion}

The incidence of somatic transformation in GCT has been described in other centres. Ahmed et al. (8) have reported 17 cases with an established diagnosis of malignant teratoma transformation out of 580 GCT patients treated with chemotherapy. Malignant mesodermal differentiation was the most common, seen in $15 / 17$ patients including rhabdomyosarcoma, leiomyosarcoma and others. Guo et al. (18) have reported 30 confirmed cases of GCT with sarcomatous component from 1985 to 2007 , all containing a teratomatous component and a sarcomatous component in the primary $(n=19)$ or metastatic tumours $(n=11)$ or both. The commonest sarcomatous component was rhabdomyosarcoma, found in $79 \%$ of cases.

The pathogenesis of somatic transformation remains unclear. It may derive from pluripotent germ cells and malignant transformation of teratomatous elements (19). There are potentially other mechanisms that contribute to the 


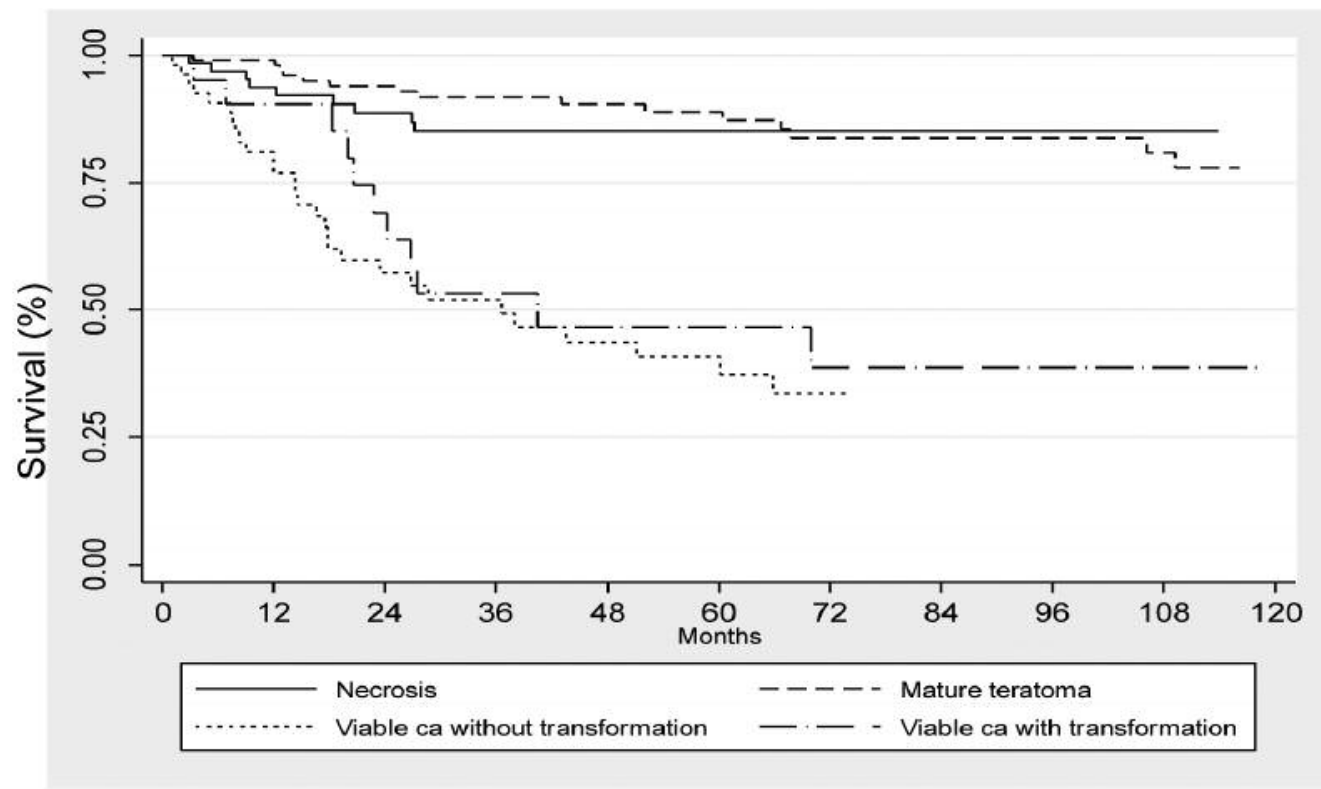

Figure 3. Survival by histology at post-chemotherapy resection. Comparison between necrosis, mature teratoma, non-sarcomatoid and sarcomatoid variants.

development of a sarcomatoid change specifically within NSGCT (non seminomatous germ cell tumour), and one hypothesis implicates exposure to DNA damaging agents. Other centres have found that the sarcoma element can appear after chemotherapy in metastasis rather than being present in the primary excision (20) and this is certainly evident in our cohort presented above. A recent, elegant, genomic study on chemotherapy resistant and refractory GCT demonstrated that loss of pluripotency markers such as NANOG is strongly associated with chemo resistance and poor outcome (21). Therefore, GCT tumours that have undergone terminal differentiation towards a specific somatic phenotype such as rhabdomyosarcoma may, in keeping with this, be expected to be more chemoresistant.

At present, it is unclear to the treating physician whether this histological diagnosis should change the therapeutic algorithm in these patients. We report on the presentation and clinical course of 30 patients with GCT that underwent somatic transformation, and find evidence for a different prognostic significance depending on the timepoint during therapy at which the somatic transformation event is seen. Despite the small number of patients, our data suggest that patients with somatic transformation at presentation appear to have a better outcome than patients who present with somatic transformation at post chemotherapy surgery for residual cancer, or at a post-chemotherapy relapse. In a retrospective study, Malagon et al. (16) have reported the clinicopathological features of 46 patients with GCT that showed additional sarcomatous components involving either the primary site or their metastases. They showed a statistically significant difference in overall survival between patients with sarcomatous containing GCT and age and stage matched control group without sarcoma in the primary site $(p<0.001)$. Guo et al. have also found a higher risk of mortality in patients with sarcoma in any metastasis (18).

Currently, there is no consensus on the treatment of GCTs with a sarcomatous component due to the rarity of this tumour. Cisplatin-based combination chemotherapy remains the backbone of treatment for disseminated NSGCTs achieving excellent anticipated cure rates in good risk patients. However, treatment of platinum refractory disease is challenging and a significant number of patients with intermediate and poor risk NSGCT will still require combination chemotherapy for relapsed disease.

In many centres, ifosfamide and platinum-containing regimens such as TIP, VIP or VeIP (4) are utilised as first line therapy of relapse, with high dose chemotherapy and stem cell rescue held in reserve for third line salvage therapy. However, none of the large prospective randomised studies utilising salvage chemotherapy have reported on whether any of the patients had variant histology. The total numbers are likely therefore to be small.

In the case series presented above, a number of patients who progressed following first-line platinum-based chemotherapy were salvaged utilising ifosfamide-containing regimens such as TIP, G-TIP, cisplatin doxorubicin and ifosfamide. Germ cell tumours are generally highly sensitive to cisplatin-based chemotherapy whilst pure sarcomas are 
generally resistant to cisplatin. Doxorubicin and ifosfamide have been the backbone of treatment of metastatic sarcoma over the last decade, and gemcitabine-docetaxel combination has recently emerged as a useful alternative in several phase II studies. Hensley et al. have found a 53\% response rate including $50 \%$ of patients previously treated with doxorubicin (22). Some pre-clinical data suggests that there seems to be a degree of synergistic activity between docetaxel and gemcitabine (23). Donadio et al. (24) administered chemotherapy, based on the specific single cell type observed in the transformed histology, to 12 GCT patients, of which 5 presented with undifferentiated rhabdomyosarcoma, and a partial response was achieved in 7 cases. The choice of salvage chemotherapy should therefore take into consideration the data above indicating that the sarcomatous element is likely to affect outcome in relapsed disease and we would suggest utilising regimens that have documented crossover efficacy in these tumours.

The studies highlighted above $(13,17,23)$ indicate that the presence of viable cancer with sarcomatoid change may confer a poorer outcome in the post chemotherapy surgery setting compared to the presence of viable nontransformed cancers. We were able to compare a cohort of patients $(n=23)$ who underwent resection of residual disease post chemotherapy for NSGCT and were found to have viable cancer (but not transformed) with the cohort of patients described above with sarcomatoid change and post chemotherapy resection $(n=8)$. Although there is the caveat of the small number of cases, the data presented in Figure 3 indicate that the presence of residual active cancer post chemotherapy confers a poor prognosiswhether sarcomatoid or not, implying that the failure of first line systemic treatment is in itself a key determinant of survival.

In these cases, it is clear that surgery with the goal of clearance of all sites of residual disease is clearly also of vital importance. Recent literature has highlighted the importance of surgical resection of residual disease in other chemotherapy "resistant" phenotypes of GCT such as late relapse (25) and this must be echoed in patients with variant histology. In our cohort patients, whenever feasible surgery was performed, including retroperitoneal lymph node dissection (RPLND), thoracotomy and craniotomy to achieve resection of residual disease. Radiotherapy was also utilised where surgery was not deemed possible.

\section{Conclusion}

Our case series illustrates that the timepoint at which somatic transformation is seen, whether this is upfront or at relapse, changes its prognosis. The presence of a somatic transformation component in testicular GCTs is generally deemed to be an adverse prognostic factor, however we see a reasonable 5-year overall survival rate $(87.5 \%)$ in patients who present with this at diagnosis, with the 5-year survival falling to $37-40 \%$ if this is seen as a post chemotherapy or at relapse. Somewhat surprisingly, the presence of viable cancer in post chemotherapy surgery denotes a poorer outcome irrespective of whether there is somatic transformation present or not.

Detecting and accurately classifying an SC in GCT is paramount as those patients on relapse may benefit more from combination chemotherapy regimens that take into account the SC component. Maximal resection of residual disease should also be considered in patients with these variants, in order to improve long-term outcomes. Within the limitations of a retrospective case series review, this case series illustrates the need for clinicians to consider the context in which the diagnosis of sarcomatous transformation is made, and if on relapse to consider offering personalised cytotoxic regimens and, critically, multimodality treatment in this highly aggressive disease.

\section{Conflicts of Interest}

The Authors have no conflicts of interest to declare regarding this study.

\section{Authors' Contributions}

CA, JS, MM, MH and AS contributed to the concept and design of the paper. CA, JS, MM, MH, DN and AS contributed to the patient care and case presentation, AG contributed to the radiological examination and SC, HS and BD analysed and interpreted the histological examination. All Authors read and approved the final manuscript.

\section{Acknowledgements}

The study was supported by Cancer Treatment Research Trust UK (CTRT) and CRUK grant to AS.

\section{References}

1 Carver BS, Serio AM, Bajorin D, Motzer RJ, Stasi J, Bosl GJ, Vickers AJ and Sheinfeld J: Improved clinical outcome in recent years for men with metastatic nonseminomatous germ cell tumors. J Clin Oncol 25(35): 5603-5608, 2007. PMID: 179985 44. DOI: $10.1200 / J C O .2007 .13 .6283$

2 Mostofi FK: Proceedings: Testicular tumors. Epidemiologic, etiologic, and pathologic features. Cancer 32(5): 1186-1201, 1973. PMID: 4148412. DOI: 10.1002/1097-0142(197311) 32:5<1186::AID-CNCR2820320527>3.0.CO;2-8

3 Tookman L, Rashid S, Matakidou A, Phillips M, Wilson P, Ansell W, Jamal-Hanjani M, Chowdhury S, Harland S, Sarwar N, Oliver T, Powles T and Shamash J: Carboplatin auc 10 for igcccg good prognosis metastatic seminoma. Acta Oncol 52(5): 987-993, 2013. PMID: 22943385. DOI: 10.3109/0284186X. 2012.714078 
4 McCaffrey JA, Mazumdar M, Bajorin DF, Bosl GJ, Vlamis V and Motzer RJ: Ifosfamide- and cisplatin-containing chemotherapy as first-line salvage therapy in germ cell tumors: Response and survival. J Clin Oncol 15(7): 2559-2563, 1997. PMID: 9215825. DOI: 10.1200/JCO.1997.15.7.2559

5 Saxman SB, Finch D, Gonin R and Einhorn LH: Long-term follow-up of a phase iii study of three versus four cycles of bleomycin, etoposide, and cisplatin in favorable-prognosis germcell tumors: The indian university experience. J Clin Oncol 16(2): 702-706, 1998. PMID: 9469360. DOI: 10.1200/JCO.1998. 16.2 .702

6 Spiess PE, Pisters LL, Liu P, Pettaway CA, Kamat AM, Gomez JA and Tannir NM: Malignant transformation of testicular teratoma: A chemoresistant phenotype. Urol Oncol 26(6): 595-599, 2008. PMID: 18367105. DOI: 10.1016/j.urolonc. 2007.07.013

7 Ulbright TM, Loehrer PJ, Roth LM, Einhorn LH, Williams SD and Clark SA: The development of non-germ cell malignancies within germ cell tumors. A clinicopathologic study of 11 cases. Cancer 54(9): 1824-1833, 1984. PMID: 6090001. DOI: 10.1016/ s0022-5347(01)64035-7

8 Ahmed T, Bosl GJ and Hajdu SI: Teratoma with malignant transformation in germ cell tumors in men. Cancer 56(4): 860863, 1985. PMID: 2990657. DOI: 10.1002/1097-0142(198508 15)56:4<860::aid-cncr2820560426>3.0.co;2-3

9 Little JS Jr., Foster RS, Ulbright TM and Donohue JP: Unusual neoplasms detected in testis cancer patients undergoing postchemotherapy retroperitoneal lymphadenectomy. J Urol 152(4): 1144-1149, 1994. PMID: 7820142.

10 Michael H, Hull MT, Ulbright TM, Foster RS and Miller KD: Primitive neuroectodermal tumors arising in testicular germ cell neoplasms. Am J Surg Pathol 21(8): 896-904, 1997. PMID: 9255252.

11 Motzer RJ, Amsterdam A, Prieto V, Sheinfeld J, Murty VV, Mazumdar M, Bosl GJ, Chaganti RS and Reuter VE: Teratoma with malignant transformation: Diverse malignant histologies arising in men with germ cell tumors. J Urol 159(1): 133-138, 1998. PMID: 9400455. DOI: 10.1016/s0022-5347(01)64035-7

12 Magers MJ, Kao CS, Cole CD, Rice KR, Foster RS, Einhorn LH and Ulbright TM: "Somatic-type" malignancies arising from testicular germ cell tumors: A clinicopathologic study of 124 cases with emphasis on glandular tumors supporting frequent yolk sac tumor origin. Am J Surg Pathol 38(10): 1396-1409, 2014. PMID: 24921638. DOI: 10.1097/PAS.0000000000000262

13 Howitt BE, Magers MJ, Rice KR, Cole CD and Ulbright TM: Many postchemotherapy sarcomatous tumors in patients with testicular germ cell tumors are sarcomatoid yolk sac tumors: A study of 33 cases. Am J Surg Pathol 39(2): 251-259, 2015. PMID: 25229769. DOI: 10.1097/PAS.0000000000000322

14 Ulbright TM, Clark SA and Einhorn LH: Angiosarcoma associated with germ cell tumors. Hum Pathol 16(3): 268-272, 1985. PMID: 2982720

15 Shamash J, Powles T, Ansell W, Berney D, Stebbing J, Mutsvangwa K, Wilson P, Asterling S, Liu S, Wyatt P, Joel SP and Oliver RT: GAMEC--a new intensive protocol for untreated poor prognosis and relapsed or refractory germ cell tumours. $\mathrm{Br}$ J Cancer 97(3): 308-314, 2007. PMID: 17609665. DOI: 10.1038/ sj.bjc. 6603865
16 Malagon HD, Valdez AM, Moran CA and Suster S: Germ cell tumors with sarcomatous components: A clinicopathologic and immunohistochemical study of 46 cases. Am J Surg Pathol 31(9): 1356-1362, 2007. PMID: 17721191. DOI: 10.1097/PAS. $0 \mathrm{~b} 013 \mathrm{e} 318033 \mathrm{c} 7 \mathrm{c} 4$

17 Bower M, Newlands ES, Holden L, Rustin GJ and Begent RH: Treatment of men with metastatic non-seminomatous germ cell tumours with cyclical POMB/ACE chemotherapy. Ann Oncol 8(5): 477-483, 1997. PMID: 9233528. DOI: 10.1023/a:10082 79222625

18 Guo CC, Punar M, Contreras AL, Tu SM, Pisters L, Tamboli P and Czerniak B: Testicular germ cell tumors with sarcomatous components: An analysis of 33 cases. Am J Surg Pathol 33(8): 1173-1178, 2009. PMID: 19561445. DOI: 10.1097/PAS.0b0 13e3181adb9d7

19 Kaw YT and Cramer HM: Cytologic diagnosis of rhabdomyosarcoma in a patient with germ cell tumor. A case report. Acta Cytol 39(2): 249-251, 1995. PMID: 7887073.

20 Terrier-Lacombe MJ, Martinez-Madrigal F, Porta W, Rahal J and Droz JP: Embryonal rhabdomyosarcoma arising in a mature teratoma of the testis: A case report. J Urol 143(6): 1232-1234, 1990. PMID: 2188021. DOI: 10.1016/s0022-5347(17)40235-7

21 Taylor-Weiner A, Zack T, O'Donnell E, Guerriero JL, Bernard B, Reddy A, Han GC, AlDubayan S, Amin-Mansour A, Schumacher SE, Litchfield K, Turnbull C, Gabriel S, Beroukhim R, Getz G, Carter SL, Hirsch MS, Letai A, Sweeney C and Van Allen EM: Genomic evolution and chemoresistance in germ-cell tumours. Nature 540(7631): 114-118, 2016. PMID: 27905446. DOI: 10.1038 /nature20596

22 Hensley ML, Maki R, Venkatraman E, Geller G, Lovegren M, Aghajanian C, Sabbatini P, Tong W, Barakat R and Spriggs DR: Gemcitabine and docetaxel in patients with unresectable leiomyosarcoma: Results of a phase II trial. J Clin Oncol 20(12): 2824-2831, 2002. PMID: 12065559. DOI: 10.1200/JCO.2002. 11.050

23 Leu KM, Ostruszka LJ, Shewach D, Zalupski M, Sondak V, Biermann JS, Lee JS, Couwlier C, Palazzolo K and Baker LH: Laboratory and clinical evidence of synergistic cytotoxicity of sequential treatment with gemcitabine followed by docetaxel in the treatment of sarcoma. J Clin Oncol 22(9): 1706-1712, 2004. PMID: 15117993. DOI: 10.1200/JCO.2004.08.043

24 Donadio AC, Motzer RJ, Bajorin DF, Kantoff PW, Sheinfeld J, Houldsworth J, Chaganti RS and Bosl GJ: Chemotherapy for teratoma with malignant transformation. J Clin Oncol 21(23): 4285-4291, 2003. PMID: 14645417. DOI: $10.1200 / \mathrm{JCO} .2003$ .01 .019

25 Baniel J, Foster RS, Gonin R, Messemer JE, Donohue JP and Einhorn LH: Late relapse of testicular cancer. J Clin Oncol 13(5): 1170-1176, 1995. PMID: 7537800. DOI: 10.1200/JCO. 1995.13.5.1170 